\title{
The Effects of $E_{2}$ Supplementation from the Early Proliferative Phase to the Late Secretory Phase of the Endometrium in hMG-Stimulated IVF-ET
}

\author{
HYUK JUNG ${ }^{1}$ and HYOUNG KYUN ROH ${ }^{1}$
}

Submitted: April 28, 1999

Accepted: June 28, 1999

Purpose: Our purpose was to determine if pregnancy rates (PRs) for hMG (human menopausal gonadotropin)-stimulated IVF-ET (in vitro ferilization-embryo transfer) can be increased by estradiol $\left(E_{2}\right)$ supplementation from the early proliferative phase to the late secretory phase of the endometrium.

Method: Eighty-one infertile women with pure tubal factor were randomized into two groups. One group received no $E_{2}$ supplementation (control group) and the other received oral $E_{2}$ supplementation ( $2 \mathrm{mg}$ two times daily) from the early proliferative phase starting on the third day of the menstrual cycle to the late secretory phase of the endometrium, with hMG stimulation for ovulation induction starting on the sixth day of the menstrual cycle.

Results: In 85 cycles, at least one embryo was transferred. Compared with the control group $(n=27$ cycles $)$, the $E_{2}$ supplementation group ( $n=58$ cycles) had a significantly higher PR (control, 25.9\%, versus $E_{2}$ supplementation, $48.3 \%$ ) and IR per ET (control, $10 \%$, versus $E_{2}$ supplementation, 26\%), but FRs per retrieved oocytes were not statistically different between the two groups (control, $74 \%$, versus $E_{2}$ supplementation group, $73 \%$ ). Four spontaneous abortions occurred in the $E_{2}$ supplementation group, and one case in the control group. Ectopic pregnancy occurred in one case in the control group.

Conclusions: Clinical PRs and IRs in the $E_{2}$ supplementation group were significantly higher than in the control group, while FRs in the control group did not differ statistically from the $E_{2}$ supplementation group. This suggests that $E_{2}$ supplementation from the early proliferative phase to the late secretory phase of the endometrium in $h M G$-stimulated IVF-ET increases the receptivity of the endometrium for transferred embryos and clinical PRs.

KEY WORDS: in vitro fertilization-embryo transfer; estradiol valerate.

\footnotetext{
${ }^{1}$ Department of Obstetrics and Gynecology, College of Medicine, Chosun University, Kwangju, Korea.
}

\section{INTRODUCTION}

Embryo implantation is an essential process in mammalian pregnancy that has recently become the focus of important scientific investigations because it presents the greatest challenge to all forms of assisted human reproduction. In fact, only about $15 \%$ of human embryos replaced in the uterus complete the initial stages of implantation (1).

So far, hormonal supports during the luteal phase after oocyte retrieval have become the focus for increasing the implantation rate (2), but it is controversial whether and how the supports could increase endometrial receptivity.

We think that a well-developed proliferative endometrium should be a prerequisite for an adequate secretory endometrium for implantation

In conventional IVF-ET (in vitro fertilizationembryo transfer) with hMG (human menopausal gonadotropin) stimulation, the endometrium proliferates rapidly under a high concentration of estrogen in a short duration, which is not similar to the hormonal change in the natural menstrual cycle and may be an important clue to low implantation rates. However, in oocyte donation, exposure of the endometrium to estrogen by estradiol valerate administration for a sufficient duration has made the endometrium more receptible to transferred embryos under the state of better proliferation and maturation of the endometrium, which is similar to the hormonal change in natural menstrual cycle and could increase the implantation rate.

We designed this study under the hypothesis that supplementation of estradiol valerate starting in the early proliferative phase for a sufficient duration, followed by hMG stimulation of ovaries, can induce adequate proliferation and maturation of the endometrium 
to increase the implantation rate, which is supported by better outcomes in IVF for oocyte donation.

\section{MATERIALS AND METHODS}

\section{Patients}

Couples with male factors, endometriosis, ovarian factors, hormonal factors, etc., except tubal factors were excluded from this study by the examination protocol (hysterosalphingogram, ultrasonography, hormonal assays, laparoscopy, etc.) of our laboratory. All of the women had pure tubal factor on the basis of a hysterosalphingogram, which was peformed 4-5 days after menstruation, and a diagnostic laparoscopy.

All 81 patients undergoing IVF-ET at the Center for Reproductive Medicine and Infertility at Chosun University Hospital from June 1, 1996, to July 31, 1998, were included and randomized into two groups: in the control group, the patients were scheduled for conventional IVF-ET programs, while estradiol valerate was given orally to the study group from the early follicuar phase to the late luteal phase or early pregnancy.

\section{Protocols}

All patients were scheduled for IVF-ET using a long protocol of GnRH agonist. Nafarelen acetate $(800 \mu \mathrm{g} /$ day; Synarel, Searle, Korea) was given intranasally, starting on the 21 st menstrual day, for pituitary desensitization. Gonadotropin therapy was begun using the combination of $\mathrm{hMG}$ and follicle-stimulating hormone using an established protocol.

In the control group, follicle stimulating hormone (FSH) (LG, Korea), 150 IU im, and hMG (LG), 150 IU im, were given daily for ovarian stimulation starting when down-regulation was achieved $\left(\mathrm{E}_{2},<30 \mathrm{pg} / \mathrm{ml}\right.$ on day 3 after uterine withdrawal bleeding) (Fig. 1). In the study group, starting 3-4 days after supplementation of oral Estradiol $\left(\mathrm{E}_{2}\right)$ valerate $(4 \mathrm{mg} /$ day $)$ had begun, hMG, $150 \mathrm{IU}$ im, and FSH, $150 \mathrm{IU}$ im, were given daily. The dosage of gonadotropins was adjusted according to the individual response.

In all of the women, daily monitoring of follicular growth was generally initiated on the third or fourth day of gonadotropin therapy. Follicular monitoring was performed with a $7.5-\mathrm{MHz}$ transvaginal probe using ultrasound.

The timing of hCG (human chorionic gonadotropin) was based on the mean lead follicular diameter. In general, hCG was administered at a dosage of 10,000 IU im when the presence of two or more follicles $>18$ $\mathrm{mm}$ in diameter were confirmed by vaginal ultrasonography. In the case that two or fewer follicles were growing, the cycle was canceled.

Gonadotropin-releasing hormone agonist administration ended in all cycles on the day of hCG injection. Oocyte aspiration was performed by vaginal ultrasonography under local anesthesia 34-36 hr after hCG administration.

Human tubal fluid (HTF; Irvine Scientific, Catalog No. 9962, USA) was used for culturing, supplemented with synthetic serum substitute (SSS; Irvine Scientific, Catalog No. 99193) and tested in our laboratory. The medium was preserved at $4^{\circ} \mathrm{C}$ before insemination.

Spermatozoa for insemination were prepared using the swim-up technique after samples from masturbation were collected. Embryos were examined for cleavage $42-46 \mathrm{hr}$ after insemination and transferred into the uterine cavity at the two- to four-cell stage.

Luteal support was given in each case where an embryo transfer took place. For luteal support, progesterone-in-oil was given intramuscularly in all of the women from the day of oocyte retrieval, starting with a dosage of $50 \mathrm{mg} /$ day, increasing to $100 \mathrm{mg} /$ day from the day of ET. Supplemental progesterone-in-oil continued until either a negative pregnancy test or sonographically confirmed embryonic viability was noted.

In the study group, oral estradiol valerate was given at a dosage of $4 \mathrm{mg} /$ day in addition to progesterone support during the luteal phase to the day when a negative pregnancy test was noted or the day when the presence of intrauterine embryonic heart activity on transvaginal ultrasonography was detected in the case of a positive pregnancy test. Only progesterone was given for luteal support in the control group.

In the case of a positive pregnancy test, progesterone for luteal support was given until 9-12 weeks gestational age.

Biochemical pregnancy was confirmed by a serum $\beta$-hCG $>10 \mathrm{IU} / \mathrm{L}$. Clinical pregnancies required at minimum the presence of a detectable gestational sac on ultrasound or the histological finding of chorionic villi in the event of miscarriage or ectopic pregnancy. Ongoing pregnancies were defined by the presence of intrauterine embryonic heart activity on transvaginal ultrasonography and were either continuing or delivered pregnancies at the time of this analysis.

Cycle cancellation was undertaken in the event of a poor response to stimulation.

Data were gathered on age, days of stimulation, endometrial thickness at the time of hCG injection, 


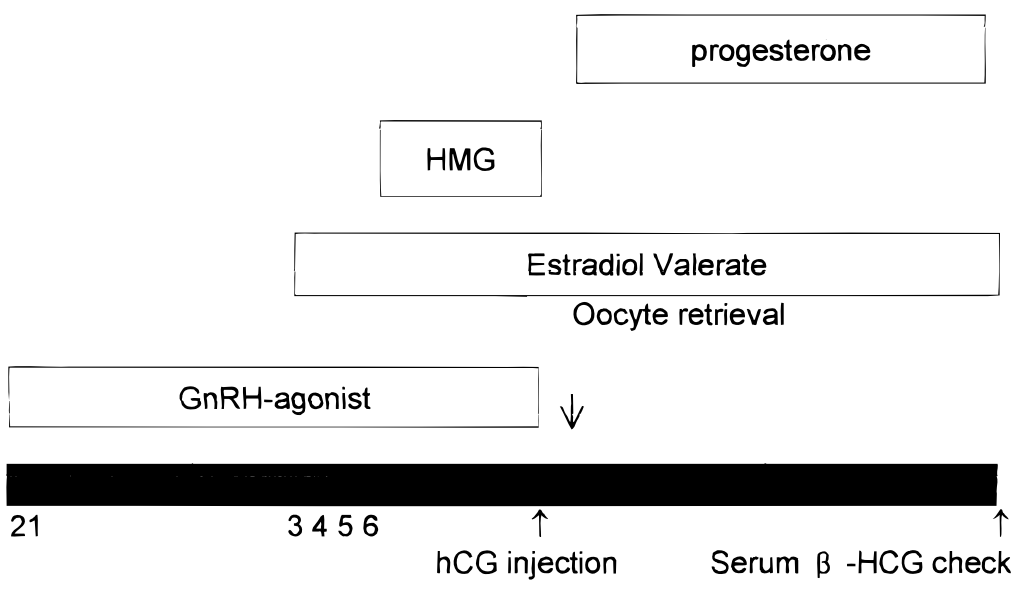

Fig. 1. Schematic feature of the protocol used in the control group.

number of oocytes retrieved and fertilized embryos transferred, clinical pregnancies, and spontaneous abortions.

\section{Statistics}

Statistical comparisons were performed by $\chi^{2}$ analysis or Student's $t$ test. $P<0.05$ was considered statistically significant. The data are expressed as the mean $\pm \mathrm{SD}$.

\section{RESULTS}

The results are summarized in Tables I-III.

As shown in Table I, the age distributions in the control and the study groups were almost-identical, and the mean age of all patients was 32.30 years. Table I also shows the data regarding ovarian response in the two groups studied.

The duration of controlled ovarian hyperstimulation $(\mathrm{COH})$ was not similar for the two groups of IVF patients. There were slightly more days in the study group (9.86 versus 8.52 days; $P<0.01)$. The endome-

Table I. Age and the Response of Ovaries and the Endometrium in Embryo Transfers

\begin{tabular}{|c|c|c|c|}
\hline & $\begin{array}{l}\text { Control } \\
\text { group } \\
(n=27)\end{array}$ & $\begin{array}{c}\text { Study } \\
\text { group } \\
(n=58)\end{array}$ & $P$ \\
\hline Age (years) & $31.29 \pm 3.71$ & $33.24 \pm 4.40$ & $>0.05$ \\
\hline Duration of $\mathrm{COH}$ (days) & $8.52 \pm 1.28$ & $9.86 \pm 1.39$ & $<0.01$ \\
\hline $\begin{array}{l}\text { Endometrial thickness on } \\
\text { day of hCG injection }(\mathrm{mm})\end{array}$ & $10.74 \pm 2.64$ & $12.03 \pm 2.81$ & $<0.05$ \\
\hline
\end{tabular}

trial thickness on the day of hCG administration was significantly higher in the study group (12.03 versus $10.74 \mathrm{~mm} ; P<0.05$ ).

Regarding ovum retrieval and IVF outcome (Tables II and III), the number of retrieved oocytes, the number of fertilized oocytes, and the number of transferred oocytes were higher in the control group, while the number of G-sacs confirmed by transvaginal ultrasound was similar for the two groups of IVF patients.

The fertilization rate (number of fertilized oocytes/ number of retrieved oocytes) was not statistically different between the two groups (74 versus $73 \% ; P>$ 0.05 ). But the implantation rate (number of G-sacs confirmed by transvaginal ultrasound/number of transferred embryos) and the clinical pregnancy rate (number of pregnant cycles confirmed by ultrasound/ number of cycles where at least one embryo was transferred) in the study group were significantly higher than in the control group (26\% versus $10 \%, P<0.05$; $48.3 \%$ versus $25.9 \%, P<0.05)$. This suggests that the endometrial receptivity was increased in the study group while the quality of retrieved oocytes was not different between the two groups.

Table II. Ovum Retrieval and Outcome of IVF

\begin{tabular}{lrrr}
\hline & $\begin{array}{c}\text { Control } \\
\text { group } \\
(n=27)\end{array}$ & $\begin{array}{c}\text { Study } \\
\text { group } \\
(n=58)\end{array}$ & $P$ \\
\hline $\begin{array}{l}\text { No. of retrieved oocytes } \\
\text { No. of fertilized oocytes }\end{array}$ & $10.59 \pm 3.75$ & $7.72 \pm 3.12$ & $<0.01$ \\
$\begin{array}{l}\text { No. of transferred embryos } \\
\text { No. of G-sacs confirmed } \\
\text { by US }\end{array}$ & $0.11 \pm 2.41 \pm 1.08$ & $3.55 \pm 2.54 \pm 1.31$ & $<0.01$ \\
\hline
\end{tabular}


Table III. Comparison of Pregnancy Outcomes

\begin{tabular}{lccc}
\hline & $\begin{array}{c}\text { Control group } \\
(n=27)\end{array}$ & $\begin{array}{c}\text { Study group } \\
(n=58)\end{array}$ & $P$ \\
\hline Fertilization rate $^{a}$ & $74 \%$ & $73 \%$ & $>0.05$ \\
Implantation rate $^{b}$ & $10 \%$ & $26 \%$ & $<0.05$ \\
Pregnancy rate $^{c}$ & $25.9 \%$ & $48.3 \%$ & $<0.05$ \\
\hline
\end{tabular}

${ }^{a}$ No. of fertilized oocytes/No. of retrieved oocytes.

${ }^{b}$ No. of G-sacs confirmed by ultrasound/No. of transferred embryos.

${ }^{c}$ No. of pregnant cycles confirmed by ultrasound/No. of cycles where at least one embryo was transferred.

\section{DISCUSSION}

Since hMG-stimulated IVF-ET was started, there has not been a significant increase in pregnancy rates despite many trials by investigators for increasing pregnancy outcomes. In past studies, the focus was on what method makes the quality of oocytes and embryos better, to increase the implantation rate. Recently, improving endometrial receptivity for the embryo is becoming the main focus. But the exact mechanism of how the embryo implants in IVF-ET is unknown, which is the main factor for increasing pregnancy outcomes; therefore, pregnancy outcomes have not changed from the past.

Maturation of the endometrium to receive embryos requires the proper hormonal change of estradiol and progesterone $(\mathrm{P})$. Estrogen proliferates endometrial cells in the basal layer and increases progesterone receptors, and progesterone induces the secretion of endometrial cells and decidualization of the stromal layer (3). Since estrogen priming is a prerequisite for the synthesis of $\mathrm{P}$ receptors in the endometrium (4), only assuring adequate $E_{2}$ and $P$ levels may allow proper endometrial maturation before implantation.

But the hormonal changes in IVF-ET differ from those in the natural menstrual cycle. Most IVF-ETs include the process of controlled ovarian hyperstimulation for the retrieval of many oocytes and serum $E_{2}$ is rapidly increased by hyperstimulated follicles in a short duration. Therfore in IVF-ET, sufficient time for maturation is not given to the endometrium to receive transferred embryos, which is very different from the natural menstrual cycle and very important in this study design. It is supposed that exposure of the endometrium to a high concentration of estrogen for a short duration during controlled ovarian hyperstimulation does not allow adequate maturation of the endometrium for implantation.

It has been reported that IVF-ET for oocyte donation has a better outcome than conventional IVF-ET gener- ally, which may be due not to the good quality of oocytes but to the administration of estrogen, which allows adequate proliferation and maturation of the endometrium for implantation of transferred embryos.

We think that a well-developed proliferative endometrium should be a prerequisite for an adequate secretory endometrium for implantation. In this study, it is suggested that hMG administration after induction by estadiol valerate supplementation to proliferate endometrium exposes the endometrium to estrogen for a sufficient period, which matures the endometrium adequately and increases the receptivity for embryos. This is based on higher pregnancy rates and implantation rates in the study group than the control group but equal fertilization rates.

Recently, vaginal ultrasonography has been used to define the endometrial thickness, which may be a predictor for implantation (5). Gonen et al. (6) reported, in donor insemination natural cycles, that pregnancy did not occur when the endometrial thickness was $<6 \mathrm{~mm}$. In addition, a similar relationship of endometrial thickness to pregnancy rate has been reported following ovulation and in vitro fertilization treatment (7-9). Others have not found any such relationship $(10,11)$

Previous data have suggested that improved prenancy rates (PRs) were seen in women with increased endometrial thickness as deterimined by sonography before oocyte retrieval. If poor PRs below a specific minimal endometrial thickness do occur, it will be interesting to note whether any therapeutic maneuvers can improve the thickness before hCG, e.g., giving hMG therapy longer. Alternatively, in patients who do not generate an appropriate endometrial thickness, it would also be interesting to determine if the cryopreservation of embryos and subsequent transfer of thawed embryos in another cycle (in which the endometrium has been primed with estrogen and progesterone after down-regulation with leuprolide acetate) may result in an increased PR. A randomized study to address these questions has been initiated (12).

In this study, the endometrial thickness of the study group was increased significantly more than that of the control group ( 12.03 versus $10.74 \mathrm{~mm} ; P<0.05$ ), which could be a factor that increases the pregnancy outcomes.

To clarify the status of the endometrium, the use of endometrial biopsy was needed. But we evaluated the endometrium non-invasively with ultrasound. Because endometrial biopsies could cause endometrial injuries, the use of endometrial biopsies for evaluation of the endometrium has the risk of abortion. It is necessary 
that endometrial biopsies to evaluate the status of the endometrium are used in future studies.

While Lewin and Smitz reported that estrogen and progesterone support during the luteal phase could not increase the PR in IVF-ET, as Ghosh and Sengupta have pointed out (13), a rise in peripherally circulating estrogen concentrations is generally observed in the midluteal phase of the menstrual cycle, and this trend is clearly sustained in conception cycles. When the normal luteal-phase pattern is presumed to be defective, following pituitary desensitization with $\mathrm{GnRH}$ analogues in conjunction with $\mathrm{hMG}$ administration for IVF and related procedures, it is common practice to support the luteal phase with hCG supplementation. This results in the restoration of elevated concentrations of both progesterone and estrogen in the midluteal phase. However, when the alternative strategy for luteal support, i.e., steroid supplementation, is employed, it is routine to omit the estrogen component and support with exogenous progesterone alone, an approach which fails to recognize a critical role for estrogen during the periimplantation period. Indeed it has been demonstrated that the inclusion of estradiol valerate during such luteal steroid supplementation does not alter the observed implantation rate compared to that achieved with the use of progesterone alone (14).

In contrast, in women with absent or nonfunctioning ovaries undergoing ET following oocyte donation, exogenous progesterone and estrogen are routinely administered during and beyond the artificial luteal phase (15). This implies the acceptance of a role for estrogen or, at least, a reluctance to accept that the available evidence rules out this possibility. The association between elevated and steadily increasing serum $\mathrm{E}_{2}$ levels after IVF-ET and higher PRs is well established (16,17).

Both $\mathrm{E}_{2}$ and $\mathrm{P}$ production by the corpus luteum plays an important role in the establishment of a pregnancy (18-20). Comparing serum $\mathrm{E}_{2}$ and $\mathrm{P}$ levels in the luteal phase of IVF-ET cycles with those in spontaneous cycles has revealed a significant decline in both hormone levels during the fifth to the ninth day after oocyte pickup, which was not observed in spontaneous cycles (21). So it was necessary for assuring $E_{2}$ and $\mathrm{P}$ levels to make the cycle resemble a spontaneous cycle and for preventing an unstable endometrium by the decline of estrogen that estradiol valerate supplementation was continued during the luteal phase and early pregnancy starting on days 3-4 before hMG administration in this study.
In conclusion, supplementation of estradiol valerate from the early proliferative phase to the late secretory phase of the endometrium in hMG-stimulated IVFET increases the receptivity of the endometrium for transferred embryos and clinical PRs. We think that $\mathrm{E}_{2}$ supplementation for a sufficient duration, starting in the early proliferative phase, can induce adequate proliferation and maturation of the endometrium and make the endometrium more receptible to transferred embryos, which increases the implantation rate and pregnancy outcomes in IVF-ET.

To make the conclusion clear, a more delicate design of the study is necessary, such as endometrial biopsy to evaluate the endometrium and serum estrogen level assay. And the results of a third group-randomized to $\mathrm{E}+\mathrm{P}$ supplementation in the luteal phase + no E supplementation in the proliferative phase-would help ascertain if one really needs estrogen supplementation in the proliferative phase.

In this study, we did not check the serum level of estrogen, did not grade the retrieved oocytes, and did not design a third group. Although there are a lot of methods to evaluate the endometrium, we performed only ultrasound to measure the thickness of the endometrium. In the future, further studies should be designed including the items pointed out to disclose the cause of the increased clinical pregnancy rate.

\section{REFERENCES}

1. Edwards RG: Clinical approaches to increasing uterine receptivity during human implantation. Hum Reprod 1995;10 (Suppl 2):60-66

2. Lewin A, Benshushan A, Mezker E, Yanai N, Schenker JG, Goshen R: The role of estrogen support during luteal phase of in vitro fertilization-embryo transplant cycles: A comparative study between progesterone alone and estrogen and progesterone support. Fertil Steril 1994;62(1):121-125

3. Buttram VC, Turati G: Uterine synechiae: Variations in severity and some conditions which may be conducive to severe adhesions. Int J Fertil 1977;22:98-103

4. Katzenellenbogen BS: Dynamics of steroid hormone receptor action. Annu Rev Physiol 1980;42:17-35

5. Gonen Y, Casper RF, Jacobson W, Blankier J: Endometrial thickness and growth during ovarian stimulation: A possible predictor of implantation in in vitro fertilization. Fertil Steril 1989; 52(3):446-450

6. Gonen Y, Calderon M, Dirnfeld M, Abramovici H: The impact of sonographic assessment of the endometrium and meticulous hormonal monitoring during natural cycles in patients with failed donor artificial insemination. Ultrasound Obstet Gynecol 1991;1:122-126

7. Glissant A, de Mouzoun J, Frydman R: Ultrasound study of the endometrium during in vitro fertilization cycles. Fertil Steril 1985;44:786-790 
8. Gonen Y, Casper RF: Prediction of implantation by the sonographic appearance of the endometrium during controlled ovarian stimulation for in vitro fertilization (IVF) J Vitro Fert Embryo Transfer 1990;7:146-152

9. Gonen Y, Casper RF, Jacobson W, Blankier J: Endometrial thickness and growth during ovarian stimulation: A possible predictor of implantation in in vitro ferilization. Fertil Steril 1989;52:446-450

10. Fleischer AC, Herbert CM, Sacks GA, Wentz AC, Entman SS, James AE: Sonography of the endometrium during conception and non conception cycles of in vitro fertilization and embryo transfer. Fertil Steril 1986;46;442-447

11. Ueno J, Oehninger S, Brzyski RG, Ascsta AA, Phiput B, Muasber SJ: Ultrasonographic appearance of the endometrium in natural and stimulation in-vitro fertilization cycles and its corration with outcome. Hum Reprod 1991;6:901-904

12. Check JH, Nowroozi K, Choe J, Dietterich C: Influence of endometrial thickness and echo patterns on pregnanacy rates during in vitro fertilization. Fertil Steril 1991;56(6):1173-1175

13. Ghosh D, Sengupta J: Another look at the issue of periimplantation oestrogen. Hum Reprod 1995;10:1-7

14. Smitz J, Bourgain C, Van Waesberghe L, Camus M, Devroey P, Van Steirteghem AC: A prospective randomized study on oestradiol valerate supplementation in addition to intravaginal micronized progesterone in buserelin and HMG induced superovulation. Hum Reprod 1993;8:40-45
15. Rosenwaks Z: Donor eggs: Their application in modern reproductive technologies. Fertil Steril 1987;47:895-909

16. Hutchinson-Williams KA, DeCherney AH, Lavy G, Kiamond MP, Naftolin F, Lunenfeld B: Luteal rescus in in vitro fertilization-embryo transfer. Fertil Steril 1990;53:485-501

17. Hutchinson-Williams KA, Lunenfeld B, Kiamond MP, Lavy G, Bouert SP, DeCherney AH: Human chorionic gonadotropin, estradiol and progesterone profiles in conception and nonconception cycles in an in vitro fertilization program. Fertil Steril 1989;52:441-445

18. Yovich JL, Stanger JD, Yovich JM, Tuvik AI, Tuznex SR: Hormonal profiles in the follicular phase, luteal phase and first trimester of pregnancies arising from in vitro fertilization. $\mathrm{Br}$ J Obstet Gynecol 1985;92:374-384

19. Lejeune B, Camus M, Deachacht J, Leroy F: Differences in the luteal phase after failed or successful in vitro fertilization and embryo replacement. J Vitro Fert Embryo Transfer 1988:3:358-386

20. Nylund L, Beskow C, Carlstrom K, Fredriceon B, Quatsfson O, Luncil NO: The early luteal phase in successful and unsuccessful implantation after IVF-ET. Hum Reprod 1990;5:40-42

21. Wang L, Warnes GM, Kirby CA, Matthews CD, Norman RJ: Luteal function associated with single, multiple and ectopic embryo implantation in natural cycles or after ovarian hyperstimulation for in vitro fertilization/gamete intrafallopian transfer. Hum Reprod 1990;5:476-480 